\title{
Non-smooth modeling in surface hardening
}

\author{
A. Sahiner* \\ Suleyman Demirel University, Department of Mathematics, 32260 Isparta, Turkey
}

Received 26 June 2014, received in revised form 30 May 2018, accepted 6 June 2018

\begin{abstract}
In this study, a traditional optimization technique Fuzzy Logic and a deterministic optimization technique filled function method are employed in the modeling of surface hardening problems in physics.

K e y w o r d s: optimization, non-smooth modeling, fuzzy sets, decision making, surface coating
\end{abstract}

\section{Introduction}

Austenitic stainless steels are used in various areas of real life, such as nuclear reactors, biomedical implants, components for chemical industries [1, $2]$. Because they have poor wear resistance, yield strength, fracture, and impact toughness, there exist lots of studies related to the improvement of mechanical properties of these materials $[3,4]$. One way is the usage of the surface coatings on these materials [5]. The use of surface coatings gives an opportunity to design a material in which the most needed properties are located. Boronizing is a thermochemical surface hardening process, and it has been used to improve the surface properties of valves, burner nozzles in the utility industry [6].

On the other hand, determining to what extent the amounts of elements in alloy affect the microhardness is one of the important questions. It has been shown in [7] that in the boronizing process different steels give different reactions and this difference arises from the amount of alloying elements. Although determining the microhardness of the alloy depending on the amounts of elements in the alloy is important in material design, there are hardly any works on this subject.

In [7], the thermochemical surface treatments and also tensile properties of four steels were discussed. Also, the influence of $\mathrm{V}$-notch on the impact properties of four different borided and unborided steels were obtained (AISI 316(0.04wt.\%C), 1040(0.35wt.\%C), 4140(0.39wt.\% C), 1045(0.40wt.\% C)).
We consider the above four materials all having different amounts of carbon, silicon, manganese, and nickel. Carbon is the main element in boronizing. First, we enrich the data by using fuzzy numbers and then employ filled function method for searching maximum microhardness of the alloy concerning some of its ingredients. For example, in our work, these ingredients are carbon and silicon.

The aim here is to look for the best amounts of the elements that make the microhardness of the alloy maximum.

Fuzzy logic starts with the concept of a fuzzy set, and fuzzy sets were first introduced by Zadeh (1965) [8]. It was not applied to control systems until the 70's due to insufficient small-computer capability before that time. Following the introduction of the theory, Zadeh began to develop modeling techniques on studies [9-11]. Zadeh noted in his paper [12] that the fuzzy logic method could have been applied effectively for the formulation and approximate solution of applicable problems, particularly in such fields as economics, management science, medicine, and biology. Then it was applied by Mamdani to control the dynamic systems in the mid-1970's. At the same study, he developed a method for controlling plants that are difficult to model [13]. After this, fuzzy logic has been widely used in many control applications, especially for nonlinear systems.

Two well-known fuzzy inference systems are Mamdani and Tagaki-Sugeno fuzzy methods [13, 14]. Mamdani method has some advantages; it is intuitive, has

*Corresponding author: e-mail address: ahmetsahiner@sdu.edu.tr 
widespread acceptance and is well suited to human cognition [15-18]. The paper [19] suggested a method of multi-dimensional fuzzy reasoning concerned with both modus ponens and modus tollens. It also discusses an example to show how the method works. In 1985, Tagaki and Sugeno presented a mathematical tool to build a fuzzy model of a system where fuzzy implications and reasoning are used. It has a quite simple form, but it may represent highly nonlinear relations as has been shown in the examples in [20].

\section{Material and methods}

In this study, the data used for modeling is from the study by Calik et al. [7]. The detailed information about how to obtain the data can be found in this article. It should be mentioned that, in the construction of fuzzy logic modeling, we have just considered the experimental data which is obtained from board zone (the surface), and transition zone. Because the effect of boronizing is very low in matrix zone, we have not taken into account the data obtained from that zone.

In the present paper, fuzzy logic modeling is employed to extend the data. Moreover, we use filled function method (FFM) to find the global maximizer of the above physical problem which has a very important role in material design.

\section{Fuzzy Logic Approach and application}

\subsection{Fuzzy Logic Approach}

A fuzzy set $A$ is defined by

$$
A=\left\{\left(x, \mu_{\mathrm{A}}(x)\right): \quad x \in X, \mu_{\mathrm{A}}(x) \in[0,1]\right\},
$$

where $X$ is the universal set, $A$ is a fuzzy subset in $X, \mu_{\mathrm{A}}(x)$ is the membership function of $A$. A fuzzy set $A$ on $\mathrm{R}$ (Real numbers) is characterized by its membership function $\mu_{\mathrm{A}}: \mathrm{R} \rightarrow[0,1]$ having properties $\mu_{\mathrm{A}}(x)=1, x \in A, \mu_{\mathrm{A}}(x)=0, x \notin A$ and $0<\mu_{\mathrm{A}}(x)<1, x \in$ partially to $A$.

Definition 1: A fuzzy set $A$ on $\mathrm{R}$ is called normal if there is at least one point $x \in \mathrm{R}$ with $\mu_{\mathrm{A}}(x)=1$.

Definition 2: A fuzzy set $A$ on $\mathrm{R}$ is convex if for any $x, y \in \mathrm{R}$ and any $\lambda \in[0,1]$, we have $\mu_{\mathrm{A}}(\lambda x+(1-\lambda) y) \geq \min \left\{\mu_{\mathrm{A}}(x), \mu_{\mathrm{A}}(y)\right\}$.

In our application the universal set $X=\mathrm{R}$ and fuzzy sets are normal, convex, upper semi continuous and have the property that $\operatorname{supp} A=\overline{\left\{x: \mu_{\mathrm{A}}(x)>0\right\}}$ is compact.

The main idea of the fuzzy set theory is: instead of determining the exact boundaries as in an ordinary set, a fuzzy set allows no sharply defined boundaries because of generalization of a characteristic function

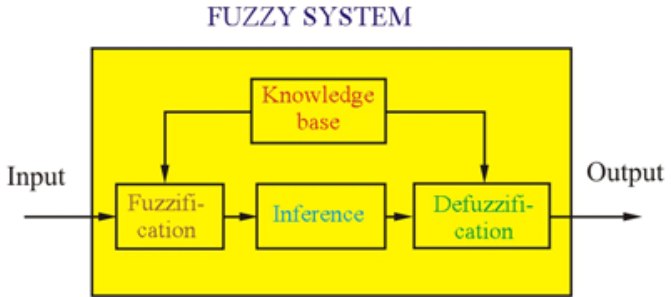

Fig. 1. The basic elements of Fuzzy Logic System.

to a membership function [21]. Figure 1 describes the basic elements of the fuzzy logic system which are rules, fuzzifier, inference engine, and defuzzifier. For any set, the degree of membership functions changes from 0 to 1 . In fuzzy logic system, there are different types of membership functions: triangular, trapezoidal, Z-shaped, Gaussian, sigmoidal, S-shaped. Triangular and trapezoidal membership functions were selected for this paper. The triangular membership function is a function of a vector $x$, and depends on three scalar parameters $a, b$, and $c$ given by

$$
\mu(x ; a, b, c)=\max \left(\min \left(\frac{x-a}{b-a}, \frac{c-x}{c-b}\right), 0\right) .
$$

The trapezoidal curve is a function of a vector $x$ and depends on four scalar parameters $a, b, c$, and $d$ as given by

$$
\mu(x ; a, b, c)=\max \left(\min \left(\frac{x-a}{b-a}, 1, \frac{d-x}{d-c}\right), 0\right) .
$$

The parameters $a$ and $d$ locate the "feet" of the trapezoid and the parameters $b$ and $c$ locate the "shoulders".

Then the linguistic rules, if then, are determined which are commands of the system behavior. After implementing these engines, the obtained results are fuzzy numbers. So they must be defuzzified. In the defuzzification stage, there are different types of methods: centroid, bisector, middle of maximum, smallest of maximum, and largest of maximum. We used centroid technique which has the formula

$$
\text { real output }=\frac{\int \mu_{\mathrm{A}}(x) x \mathrm{~d} x}{\int \mu_{\mathrm{A}}(x) \mathrm{d} x},
$$

where $\int \mu_{\mathrm{A}}(x) \mathrm{d} x \neq 0$ for all $\mu_{\mathrm{A}}$.

Because collecting the data is very costly and time consuming we use the fuzzy logic model to enrich the data.

\subsection{Application of Fuzzy Logic}

The use of fuzzy logic approach provides a basic way for the estimation of layer microhardness. Firstly, 

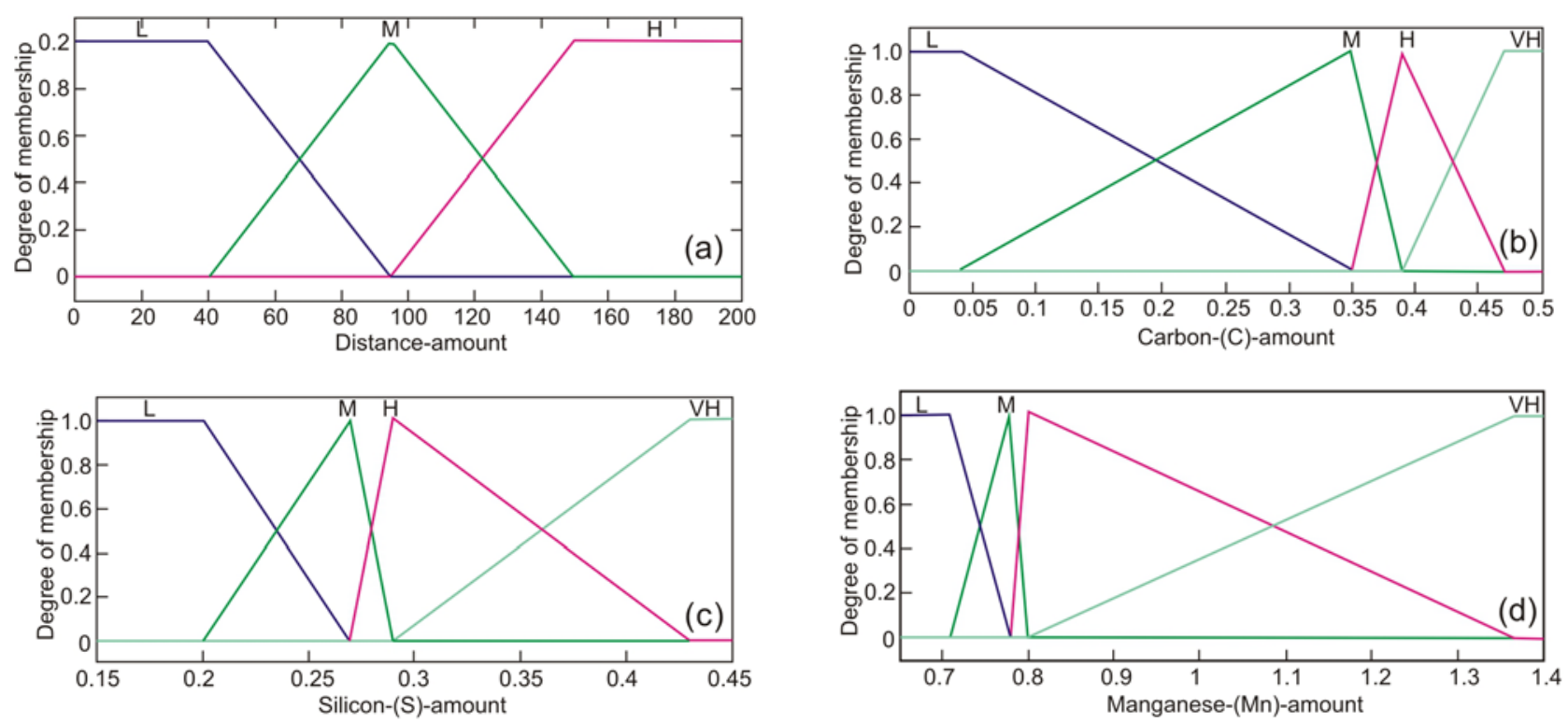

Fig. 2. (a) The membership functions of distance, (b) the membership functions of amounts of carbon, (c) the membership functions of amounts of silicon, and (d) the membership functions of amounts of manganese.

fuzzy logic modeling was used to build a Mamdani model for the estimation of layer microhardness values based on the boriding process at different amounts of carbon, silicon, manganese, and distance of boronized layer to the surface.

The amounts of carbon, silicon, manganese, a distance of boronized layer to the surface and microhardness values are selected as input and output variables, respectively. The fuzzy subsets defined for the distance of boronized layer to the surface, the amounts of carbon, silicon, and manganese are shown in Figs. 2a-d.

The distance of boronized layer to the surface, the amounts of carbon, silicon, and manganese may be defined in a scale that consist of the range $0-200 \mu \mathrm{m}$, 0-0.5 wt.\%, 0.15-4.5 wt.\%, and 0.7-1.4 wt.\%, respectively. Three fuzzy subsets, namely low, medium, and high were considered for the distance, and four fuzzy subsets, namely low, medium, high, and very high were considered for the amounts of the alloying elements carbon, silicon, and manganese. Five fuzzy subsets for the microhardness are also labeled as low, medium, high, very high, very very high (see Fig. 3).

The rule structure is designed based on how the experts interpret the characteristics of the variables of the system. In collaboration with the experts conducting the experiment, fuzzy rules of our model are obtained after considering the relationships between the input and output variables. We adjust the fuzzy partition on the computer until the best fit is obtained after the fuzzy rule base inference machine is set up. Some of the rules obtained as mentioned above are as follows:

If (Distance is L) and (Amount of carbon is L) and (Amount of silicon is VH) and (Amount of manganese

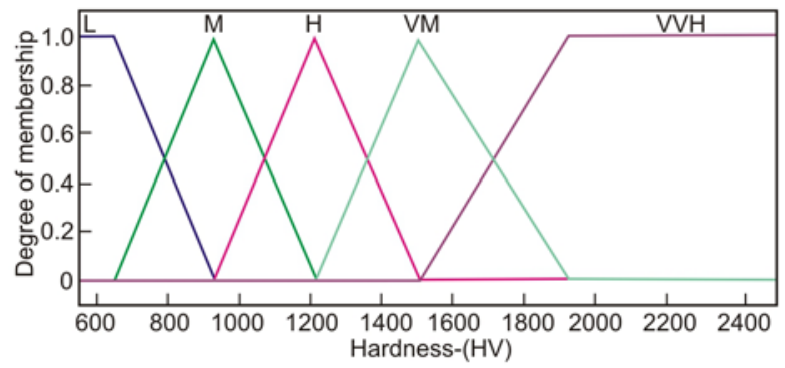

Fig. 3. The membership functions of microhardness.

is $\mathrm{VH}$ ) then (Microhardness is $\mathrm{VH}$ ).

If (Distance is $\mathrm{M}$ ) and (Amount of carbon is M) and (Amount of silicon is L) and (Amount of manganese is $\mathrm{H}$ ) then (Microhardness is $\mathrm{H}$ ).

If (Distance is $\mathrm{H}$ ) and (Amount of carbon is $\mathrm{L}$ ) and (Amount of silicon is $\mathrm{VH}$ ) and (Amount of manganese is $\mathrm{VH}$ ) then (Microhardness is $\mathrm{L}$ ).

Then a model is established which estimates the microhardness of boride layer for untested conditions by using Mamdani method. The general structure of the model is shown in the following.

In Fig. 5, the final results in the form of defuzzified microhardness values estimations versus the measured values are presented.

\section{Filled Function Method}

The Filled Function Method (FFM) which is one of the global optimization techniques was first proposed by Ge [22-25]. The first proposed two-parameter filled 


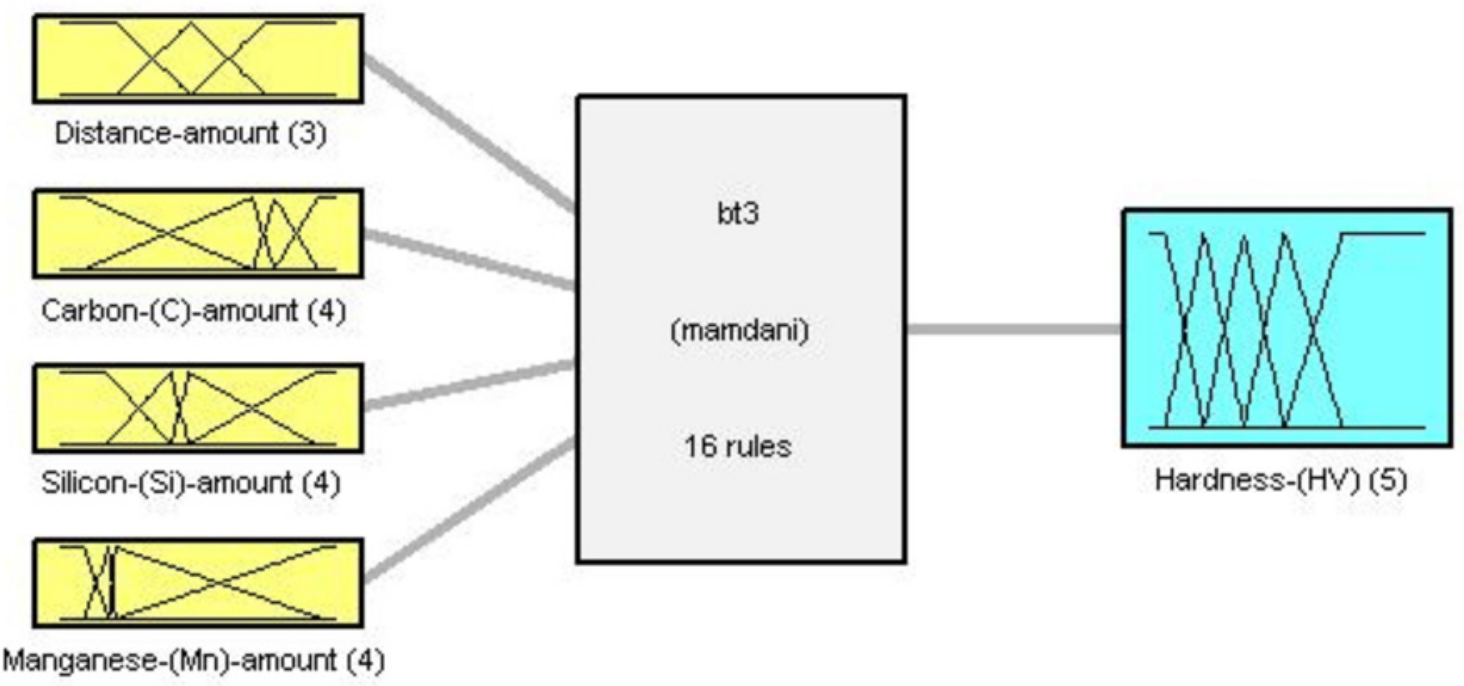

Fig. 4. Fuzzy Logic Model structure of our problem.



Fig. 5. Fuzzy values versus measured values.

function for smooth optimization is the following:

$$
P\left(x, x_{1}^{*}, r, \rho\right)=\frac{1}{r+f(x)} \exp \left(\frac{\left\|x-x_{1}^{*}\right\|^{2}}{\rho^{2}}\right) .
$$

Since it contains an exponential term, this filled function cannot be useful for every problem. Since the exponential term increases rapidly, changes in $P\left(x, x_{1}^{*}, r, \rho\right)$ and $\nabla P\left(x, x_{1}^{*}, r, \rho\right)$ become indistinguishable [22]. To avoid these drawbacks, some new filled functions were proposed in [26]. In literature, it is possible to see different studies which are trying to eliminate these drawbacks and disadvantages [27, 28]. The main idea of FFM is to leave current local minimizer $x_{1}^{*}$ to a lower minimizer $x_{2}^{*}$ of the objective function with the aid of the filled function which is constructed by modifying the objective function at the point $x_{1}^{*}$. If a local minimizer $\bar{x}$ of the filled function can be found, which lies in a basin of objective function lower than the basin of the first local minimizer, one then can find a lower minimizer $x_{2}^{*}$ of the objective function by minimizing the objective function with initial point $\bar{x}$. Just replacing $x_{1}^{*}$ by $x_{2}^{*}$, a new filled function can be constructed and then a much lower minimizer of the objective function can be found by using the same way. This loop continues until the global minimizer of the objective function is found [29-33].

Although, the first filled functions have some disadvantages and very specifically, after the development of the filled functions, the FFM has become more popular in the global optimization techniques because of its relatively easy actualization with a process similar to the sequential unconstrained minimization technique. Some definitions and details on FFM are given in the following section.

The FFM is interested in determining the global minimizer of a function $f$ on $\mathrm{R}^{n}$ under the following assumptions:

$-f$ is continuously differentiable,

- $f$ has only a finite number of minimizers,

$-f(x) \rightarrow \infty$ as $\|x\| \rightarrow \infty$.

Notice that assumption (ii) only requires that the number of local minimal values of $f$ is finite. The number of local minimizers can be infinite. Now, let us give the basic definitions related to the filled function method.

Definition 3: The basin $B_{k}^{*}$ at a local minimizer $x_{k}^{*}$ of $f(x)$ is a connected domain which contains $x_{k}^{*}$ and in which starting from any point steepest descent trajectory converges to $x_{k}^{*}$, but outside which the steepest descent trajectory does not converge to $x_{k}^{*}$.

Definition 4: A hill of $f(x)$ at $x_{k}^{*}$ is the basin of $-f(x)$ at its minimizer $x_{k}^{*}$ if $x_{k}^{*}$ is a maximizer of $f(x)$.

Definition 5: A local minimizer $x_{k+1}^{*}$ of $f(x)$ is said to be lower (higher) than $x_{k}^{*}$ if $f\left(x_{k+1}^{*}\right)<$ 
$f\left(x_{k}^{*}\right) \quad\left(f\left(x_{k+1}^{*}\right)>f\left(x_{k}^{*}\right)\right)$ and in this case the basin $B_{k+1}^{*}$ of $f(x)$ at $x_{k+1}^{*}$ is said to be lower (higher) than $B_{k}^{*}$.

Definition 6: $p(x)$ is called a filled function of $f(x)$ at a local minimizer $x_{k}^{*}$ if $p$ has the following properties:

(i) $x_{k}^{*}$ is a local maximizer of $p(x)$,

(ii) $p(x)$ has neither minimizer nor saddle point in $W_{k}^{*}-\left\{x_{k}^{*}\right\}$ and set $W_{k}^{*}$ becomes a part of $p(x)$ at $x_{k}^{*}$, where $W_{k}^{*}=B_{k}^{*} \cup U_{k}^{*}$ and $U_{k}^{*}$ is the union of all basins higher than $B_{k}^{*}$,

(iii) If $f(x)$ has a basin $B_{k+1}^{*}$ lower than $B_{k}^{*}$, then there exists a point $x^{\prime \prime} \in B_{k+1}^{*}$ that minimizes $p(x)$ along the ray $x_{k}^{*}+\lambda\left(x^{\prime \prime}-x_{k}^{*}\right), \lambda>0$ for each $x^{\prime \prime} \in$ $B_{\mathrm{k}+1}^{*}$.

The above properties of a filled function ensure that when any of descent methods, for example steepest descent method, the Newton method, the conjugate gradient method, etc., is employed to minimize the filled function, the sequence of iteration will not terminate at any point in $W_{k}^{*}$ and when there exist basins of filled function lower than $B_{k}^{*}$, the sequence will either terminate at a point in a basin lower than $B_{k}^{*}$ or generate a point $x_{s}$ such that the value of $f\left(x_{s}\right)$ is less than $f\left(x_{1}^{*}\right)$.

Using the fuzzy logic, we modeled the relation between inputs and output. In fact, the fuzzy logic approach sets the continuous function between input variables and output. If we would like to know the best values of inputs that give the maximum value of the output, unfortunately, the fuzzy logic approach does not give us the rule of the objective function so that we can find the global maximum. Therefore, some global optimization methods which need the rule of the objective (model) function cannot be applied. So, to obtain the rule and the graph of the function, we employed one of the surface fitting techniques. But for the sake of clarity, we used two inputs and one output, for example, in this work Amounts of carbon and Amounts of silicon are selected as inputs, and the microhardness is selected as the output. The rule and the graph of the model (objective function) are obtained as follows:

$$
\begin{gathered}
z=f\left(x_{1}, x_{2}\right)=\frac{1}{2}\left(4432 x_{1}^{2}-1316 x_{1}-\right. \\
\left.-253.1 \cos \left(30.38 x_{2}\right)-227.5 \sin \left(30.38 x_{2}\right)+3577\right) .
\end{gathered}
$$

The graph of a function $f$ is presented in Fig. 6 .

\section{Algorithm and application of FFM}

Insofar we have enriched data by applying fuzzy logic and constructed the objective function by using the enriched data. Herein, to find the global maximum of the objective function we give the FFM algorithm

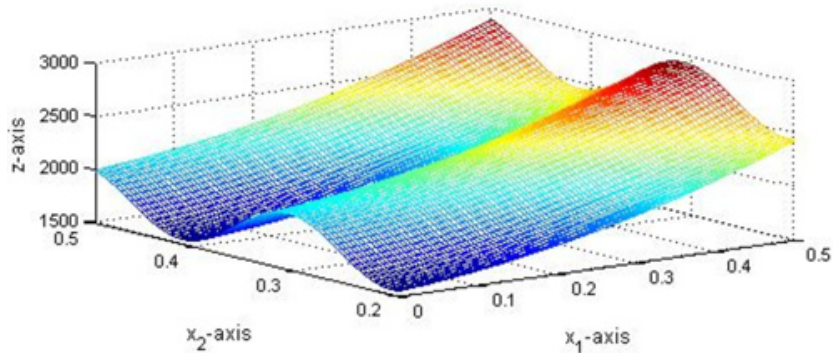

Fig. 6. The graph of the model function.

for the filled functions

$$
Q\left(x, x_{1}, a\right):=-\left(f(x)-f\left(x_{1}\right)\right) \exp \left(-a\left\|x-x_{1}\right\|^{2}\right)(7)
$$

and

$$
\begin{aligned}
S\left(x, x_{1}, a\right): & =-\left\|x-x_{1}\right\|+\mu \max \left\{0, f(x)-f\left(x_{1}\right)\right\}+ \\
& +\eta\left(\min \left\{0, f(x)-f\left(x_{1}\right)\right\}\right),
\end{aligned}
$$

where $\eta$ is mitigator, for example $\eta(t)=\arctan t$, $\eta(t)=\ln (1+t)$, proposed in [26] and [31], respectively. Notice that the function $S\left(x, x_{1}, a\right)$ is not differentiable and we need strong subgradient methods to minimize it.

Step 1. Choose a constant $\varepsilon>0$. Describe lower and upper bound for a boundary $D$ in which the global optimum of $f(x)$ is to be found. Start from any $x_{0}$ point which is within the lower and upper bounds. Minimize $f(x)$ by using a local algorithm to find a local minimizer $x_{1}$.

Step 2. Construct the filled function $Q$ (or $S$ ) by using $x_{1}$ and parameter $a$. Minimize the filled function with $x_{0}$ as a starting point to obtain a local minimizer denoted $x_{s}$.

Step 3.

- If such a point $x_{s}$ is found that it is minimizer of the function $Q$ (or $S$ ), then go to Step 4 .

- If the iteration sequence reaches the boundary of $D$, then stop and the current $x_{1}$ is adopted as the global minimizer.

Step 4. With $x_{s}$ as the starting point, minimize $f(x)$ to find another local minimizer $x_{2}\left(\neq x_{1}\right)$ (if exists).

- If $f\left(x_{2}\right)<f\left(x_{1}\right)$, update $x_{1}$ as $x_{2}$, then go to Step 2.

- If $f\left(x_{2}\right)>f\left(x_{1}\right)$, leave $x_{1}$ unchanged but update $a$ (replaced by $a+4$ ), then go to Step 2 .

After implementing the above algorithm, we obtain the following results concerning $Q$ and $S$ filled functions:

- If we use the filled function $Q$, then $x^{*}=$ $(0.5,0.334)$

- If we use the filled function $S$, then $x^{*}=$ $(0.5,0.333)$. 
We note that results are very close to each other satisfactorily. This shows our methodology starting with Fuzzy Logic to make the data continuous and modeling this data by a smooth function and ending with applying FFM to obtain the global minimizer of model function is very effective and fruitful.

\section{Conclusions and future works}

We applied fuzzy logic to estimate the microhardness of boronized steels which depends on the alloying elements. It can be used to predict the microhardness for inexperienced conditions. The regression analysis $R^{2}$ shows that experimental results and Mamdani method results are close to each other at the rate of $97.75 \%$ (see Fig. 5). The objective function which represents the model is obtained after getting useful information from enriched data. It is possible to consider different elements in modeling. In this work, we try to model the effect of the amount of alloying elements which are amount of carbon (C) and silicon (Si) on microhardness. Finally, local and global extremum values of the objective function are obtained by applying filled function method which is one of the effective global optimization techniques, that has recently been started to be applied to these kinds of physical problems (see [29-31]).

In future works, several investigations are described as follows: The same method can also be applied to some mechanical properties of alloys such as fracture toughness, residual stresses etc.; different binary alloying elements can be chosen and same method can be applied; a new filled function can be offered and the application area in engineering and physics can be extended.

Finally, it should also be noted that this new methodology can solve much more complicated physical and engineering problems whose model functions may have infinitely many local minimizers with satisfactory results. That is what we aim in our future studies.

\section{References}

[1] Cozza, R. C., de Mello, J. D. B., Tanaka, D. K., Souza, R. M.: Wear, 263, 2007, p. 111. doi:10.1016/j.wear.2007.01.099

[2] Sen, U., Sen, S.: Materials Characterization, 50, 2003, p. 261. doi:10.1016/S1044-5803(03)00104-9

[3] Dearnley, P. A., Aldrich-Smith, G.: Wear, 256, 2004, p. 491. doi:10.1016/S0043-1648(03)00559-3

[4] Shi, L., Northwood, D. O.: Acta Metallurgica et Materialia, 43, 1995, p. 453. doi:10.1016/0956-7151(94)00279-Q

[5] Ozbek, I.: Bindal, C.: Surface and Coatings Technology, 154, 2002, p. 14. doi:10.1016/S0257-8972(01)01409-8
[6] Mann, B. S.: Wear, 208, 1997, p. 125. doi:10.1016/S0043-1648(96)07374-7

[7] Calik, A., Sahin, O., Ucar, N.: Acta Physica Polonica A, 115, 2009, p. 694.

[8] Zadeh, L. A.: Information and Control, 8, 1965, p. 338. doi:10.1016/S0019-9958(65)90241-X

[9] Zadeh, L. A.: Information Sciences, 8, 1975, p. 199.

[10] Zadeh, L. A.: Information Sciences, 8, 1975, p. 301.

[11] Zadeh, L. A.: Information Sciences, 9, 1976, p. 43.

[12] Zadeh, L. A.: IEEE Transactions on Systems, Man and Cybernetics, 3, 1973, p. 28. doi:10.1049/piee.1974.0328

[13] Mamdani, E. H.: Proceedings of the Institution of Electrical Engineers, 121, 1974, p. 1585. doi:10.1049/piee.1974.0328

[14] Takagi, T., Sugeno, M.: IEEE Transactions on Systems, Man, and Cybernetics, 15, 1985, p. 116. doi:10.1109/TSMC.1985.6313399

[15] Mamdani, E. H., Assilian, S.: International Journal of Man-Machine Studies, 7, 1975, p. 1. doi:10.1016/S0020-7373(75)80002-2

[16] Esragh, F. Mamdani, E. H.: International Journal of Man-Machine Studies, 11, 1981, p. 501. doi:10.1016/S0020-7373(79)80040-1

[17] Mamdani, E. H.: IEEE Transactions on Computers, 26, 1977, p. 1182. doi:10.1109/TC.1977.1674779

[18] Saltan, M., Saltan, S., Sahiner, A.: Construction and Building Materials, 21, 2007, p. 1406. doi:10.1016/j.conbuildmat.2006.07.004

[19] Sugeno, M., Takagi, T.: Fuzzy Sets and Systems, 9, 1983, p. 313. doi:10.1016/S0165-0114(83)80030-X

[20] Sakawa, M.: Fuzzy Sets and Interactive Multi-Objective Optimization. New York, Plenum Press 1993.

[21] Kosko, B.: Neural Network and Fuzzy Systems. A Dynamical Systems Approach to Machine Intelligence. New Jersey, Prentice Hall 1992.

[22] Ge, R. P.: Mathematical Programming, 46, 1990, p. 191. doi:10.1007/BF01585737

[23] Ge, R. P.: Journal of Computational Mathematics, 5, 1987, p. 1.

[24] Ge, R. P.: Applied Mathematics and Computation, 35, 1990, p. 131. doi:10.1016/0096-3003(90)90114-I

[25] Ge, R. P., Qin, Y. F.: Journal of Optimization Theory and Applications, 54, 1987, p. 241. doi:10.1007/BF00939433

[26] Liu, X.: Applied Mathematics and Computation, 133, 2002, p. 375. doi:10.1016/S0096-3003(01)00248-X

[27] Liu, X.: Journal of Global Optimization, 19, 2001, p. 151. doi:10.1023/A:1008330632677

[28] Xu, Z., Huang, H. X., Pardalos, P., Xu, X. C.: Journal of Global Optimization, 20, 2001, p. 49. doi:10.1023/A:1011207512894

[29] Sahiner, A., Yilmaz, N., Demirozer, O.: International Journal of Pest Management, 60, 2014, p. 232. doi:10.1080/09670874.2014.958879

[30] Sahiner, A., Gokkaya, H., Yigit, T.: AIP Conf. Proc., 1479, 2012, p. 972. doi:10.1063/1.4756304

[31] Sahiner, A., Ucar, N., Yilmaz, N.: Oxidation Communications, 38, 2015, p. 166.

[32] Shang, Y., Pu, D., Jiang, A.: Applied Mathematics and Computation, 191, 2007, p. 176. doi:10.1016/i.amc.2007.02.074

[33] Yang, Y., Shang, Y.: Applied Mathematics and Computation, 173, 2006, p. 501. doi:10.1016/j.amc.2005.04.046 